\title{
Structural and capacitive characterizations of high temperature nitrogen annealed graphene oxide
}

\author{
A.N.Fouda ${ }^{1}$, M.K.Abu-Assy ${ }^{1}$ and NehadYousf ${ }^{1}$ \\ ${ }^{I}$ (Physics department, Faculty of science/ Suez canal University,41522 Ismailia, Egypt)
}

\begin{abstract}
The synthesized graphene oxide (GO) by modified hummer method was subsequently reduced by thermal annealing in nitrogen ambient at $950^{\circ} \mathrm{C}$. A perfect hexagonal GO structure was confirmed by high resolution transmission electron microscope (HRTEM) images. After thermal reduction, few layers of crystalline nitrogen annealed graphene oxide (NAGO) with dimension of hundred nano meters were obtained. The electrochemical properties of the graphene nano sheets as electrod material for supercapacitors were studied by cyclic voltammetry and electrochemical impedance spectroscopy (EIS). The area of the CV curve for the NAGO electrode is larger than those for the GO electrode indicating higher specific capacitance.
\end{abstract}

Keywords: Graphene oxide, nitrogen annealing, thermal reduction, cyclic voltammtery, supercapacitor

\section{Introduction}

Graphene is one of the most exciting topics of research in the last four to five years. Graphene is a monolayer of carbon atoms arranged in hexagonal honeycomb structure which make it the thinnest known material. Nowadays, many researchers synthesis graphene by several techniques including chemical vapor deposition [1-3], micromechanical exfoliation [4], thermal expansion [5], and reduction from GO [6-8]. Obtaining graphene by reduction of graphene oxide has the advantage of controlling the amount of oxygen according to the experimental conditions and characteristics of the parent graphite [9-10].

In addition, the reduced graphene derived from GO can not only be prepared over large scale, but also shows high mobility, large surface area and good conductivity for supercapacitors. Many attempts have been made to develop efficient approaches for the reduction of GO, by green tea, bacterial respiration, baker's yeast and carrot root [11-14]. Through their efforts, it is verified that graphene can be reduced for large scale mass production.

Recently, thermal reduction of graphene is an effective mean to fabricate graphene sheet based electrochemical double layer capacitors (EDLC). Thermally exfoliated GO is desirable because of: (i) its scalability; (ii) its sustainability, since it avoids the use of chemicals; (iii) TRGO has higher specific surface area with less agglomeration [15], and (iv) its simplicity since the exfoliation of the GO and its reduction to graphene occurs all in one step. Moreover, the graphene like materials obtained by thermal reduction are electrically conducting, thus it can be used in electrical transport applications.

Nitrogen has a comparable atomic radius to that of Oxygen. Thus, Nitrogen is theoretically predicted to substitute O,since it can fit well in the O site [16].Theoretically, Michael J. McAllister, et al. [17] postulated that a pressure of only $2.5 \mathrm{Mpa}$ is necessary to separate two stacked graphene oxide platelets. The high temperature nitrogen gas creates the required enormous pressure within the stacked layers.Recently, Bing Zhao, et al. [18] illustrated that with increasing the annealing temperature of nitrogen annealed graphene, the structural characterization of graphene were enhanced and their best result were at $900^{\circ} \mathrm{C}$. In this work, we concern in high temperature nitrogen annealing of a superior typically hexagonal GO nano sheets at $950^{\circ} \mathrm{C}$.

\section{II.1 Go Pereparation}

\section{Experimental}

GO nano sheets were prepared by a modified Hummers method [19]. Typically, 1.0g natural graphite powder and $0.5 \mathrm{~g}$ sodium nitrate were mixed with $23 \mathrm{~mL}$ sulfuric acid in an ice bath. $3 \mathrm{~g}$ potassium permanganate was slowly added and the stirring was continued for $2 \mathrm{~h}$. The mixture solution was transferred to water bath at $35^{\circ} \mathrm{C}$ and stirred for 30min. After that, $46 \mathrm{~mL}$ of deionized (DI) water was slowly added, the temperature was risen to about $98^{\circ} \mathrm{C}$. The mixture was maintained at this temperature for 30min. Successively, $140 \mathrm{ml}$ of DI water were added and $10 \mathrm{ml}$ of $\mathrm{H}_{2} \mathrm{O}_{2}$ aqueous solution (30\%). The obtained yellow suspension of $\mathrm{GO}$ was washed with diluted hydrochloric acid (5\%) and with plenty of DI water. A brown graphite oxide powder was obtained by air draying at $60^{\circ} \mathrm{C}$. To get graphene, GO sheets $0.5 \mathrm{~g}$ were thermally reduced using quartz tube furnace at $950^{\circ} \mathrm{C}$ in nitrogen ambient, the sample was named (NAGO). 


\section{II.2 Charactrization}

XRD of the samples were determined by Philips X-ray diffractometer (Model X-Pert). It utilized monochromatic $\mathrm{CuK}_{\alpha}=1.5406 \AA$ radiation which operated at $40 \mathrm{kV}$ and $25 \mathrm{~mA}$. High resolution transmission electron microscopy (HRTEM) images were recorded using Model JEM 1230, (JEOL, Japan) to observe the nano scale structures. Cyclic voltammetry measurements were done using biologic science instrument VSP.

\section{III.1material Charactrization}

\section{Result And Discussion}

XRD of graphite, GO and NAGO are shown in Fig. 1. Very sharp and high intensity (002) peak near $2 \theta=26.5^{\circ}$ was observed for graphite sample. GO nano particles shows (002) diffraction peak at $2 \theta=10.4^{\circ}$ which is mainly due to the oxidation of graphite and exfoliation process. The disappearance of the peak at $26.5^{\circ}$ and appearance of it at $10.4^{\circ}$ indicate that graphite is completely oxidized. For NAGO, no clear peak were observed in XRD while electron diffraction image confirms the crystallinity of NAGO as shown in Fig. 2f.

Fig. 2 represents HRTEM images of GO and NAGO nano sheets. A few identified hexagonal sheets were clearly observed in GO nanosheets and few layers of NAGO with dimension of few hundreds nano meters were obtained. Nitrogen annealing produced pressures which overcome the Van der Waals forces that keep the graphene sheets together.

\section{III.2electrochemical Charactrization}

Fig. 3 (a) displays the cyclic voltammogram (CV) of NAGO at different scan rates. With increasing the scan rate, a relative increment in current were found, which constitutes an ideal capacitive behavior. The same behavior was appeared for GO. No peaks were observed at different scan rates in the CV curves for all the samples, proving that the electrode is charged and discharged at a pseudo-constant rate over the complete cycle. In addition, a considerable, normal increase in the current with increasing the number of cycles was reported, as shown in Fig. 3 (b). Fig. 4 shows that NAGO current is obviously greater than GO current, which reflects the electro-catalytic activity of NAGO on the electrode surface, because of the expected increase in surface area of NAGO. Moreover, the area of the CV curve for the NAGO electrode is larger than those for the GO electrode indicating the increment of specific capacitance.

The maximum specific capacitance of the super capacitor electrodes can be calculated through equation (1) to confirm the previously mentioned statement:

$$
C_{s p}=\frac{d q}{m \cdot d V}=\frac{d q / d t}{m \cdot d V / d t}=\frac{I}{m \cdot d V / d t}
$$

Where, $\mathrm{C}_{\mathrm{sp}}$ is specific capacitance of the electrodes, $I$ is the current, $\mathrm{dV} / \mathrm{dt}$ is the rate of potential, and $m$ is the mass of the active layer on the electrodes [20]. The calculated values are plotted in Fig. 5. For different scan rates, the capacitance of NAGO shows higher values in compared with GO except at 10mV/s. The specific capacitance decreases with increasing the scan rate from $10 \mathrm{mV} / \mathrm{s}$ to $100 \mathrm{mV} / \mathrm{s}$. Such trend can be attributed to the presence of inner active sites by ion diffusion which cannot sustain the redox transitions. At higher scan rates, parts of the electrode surface are inaccessible which gives the decreasing trend.

To examine the fundamental behavior of electrode materials for supercapacitors, the electron impedance spectroscopy (EIS) analysis was done. (EIS) measurements were carried out between $200 \mathrm{kHz}$ and $0.1 \mathrm{~Hz}$. As shown in Fig. 6. Because of the faster electron transfer rate, NAGO exhibited lower impedance than GO nanosheets. Increasing in the surface morphology, high surface area, and inherent good electronic conductivity of NAGO enhance the charge transfer. The absence of Warburg curve in Fig. 6 indicates that the electrodes have short ion diffusion path, which makes the access of electrolyte ions to the surfaces of the electrodes more efficient [21].

\section{IV. $\quad$ Figures And Tables}

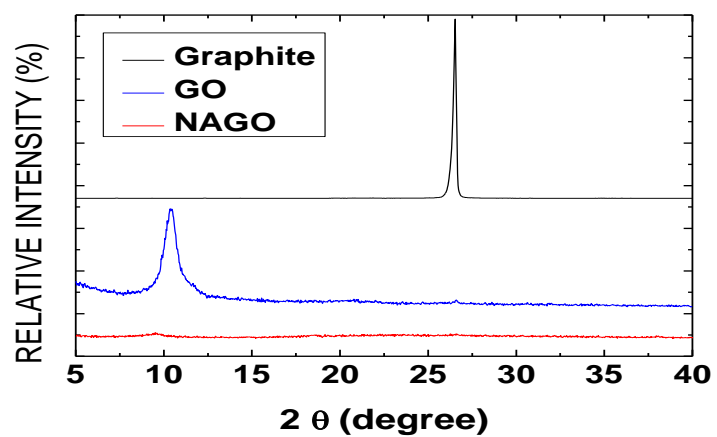

Figure 1 XRD spectra of graphite, GO and NAGO. 

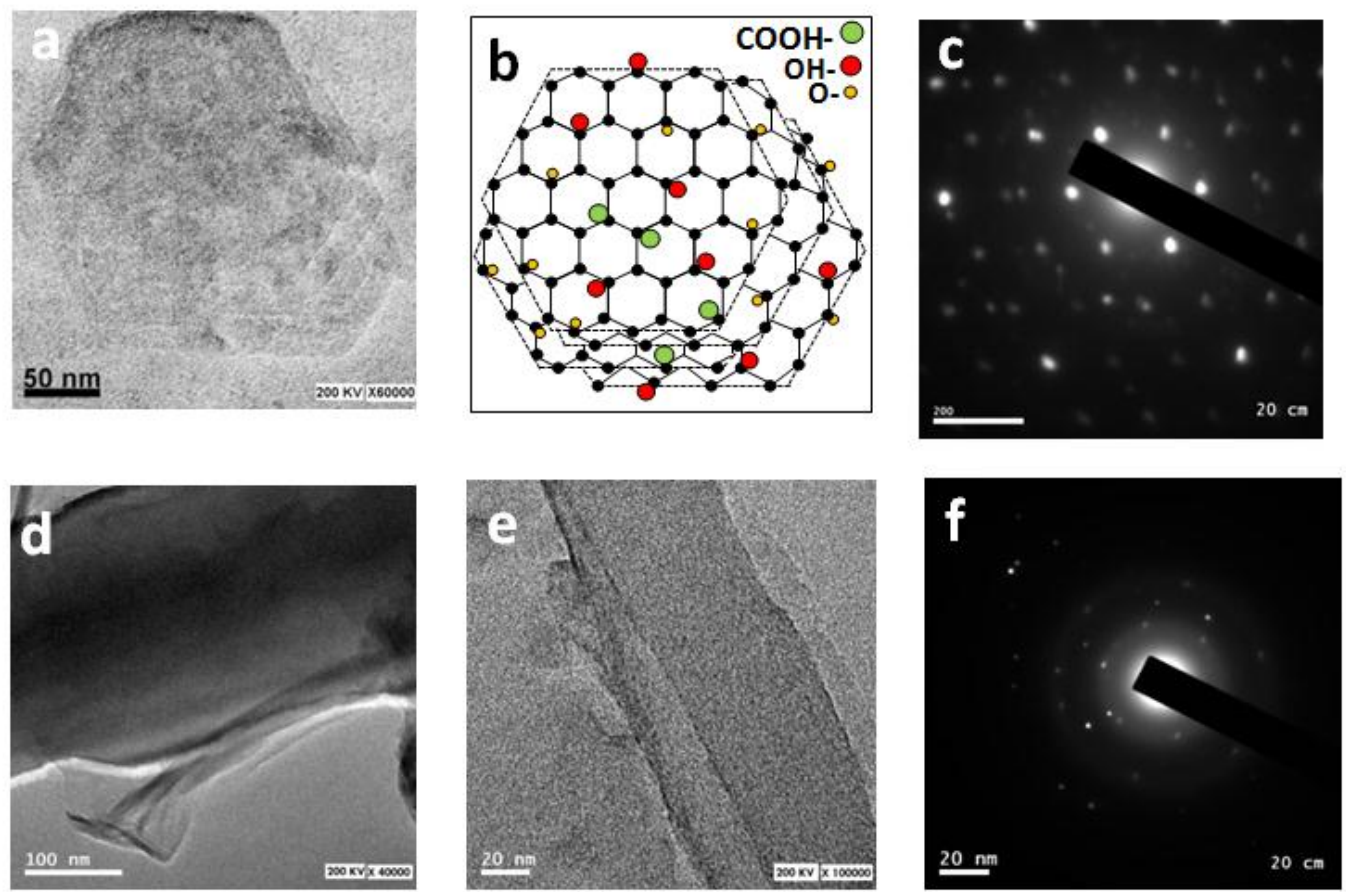

Figure 2 (a) HRTEM for GO (b) graphical representation of GO sheets (a), (d) Transmission Electron Diffraction (TED) of GO, (d, e) HRTEM of NAGO, (f) TED of NAGO
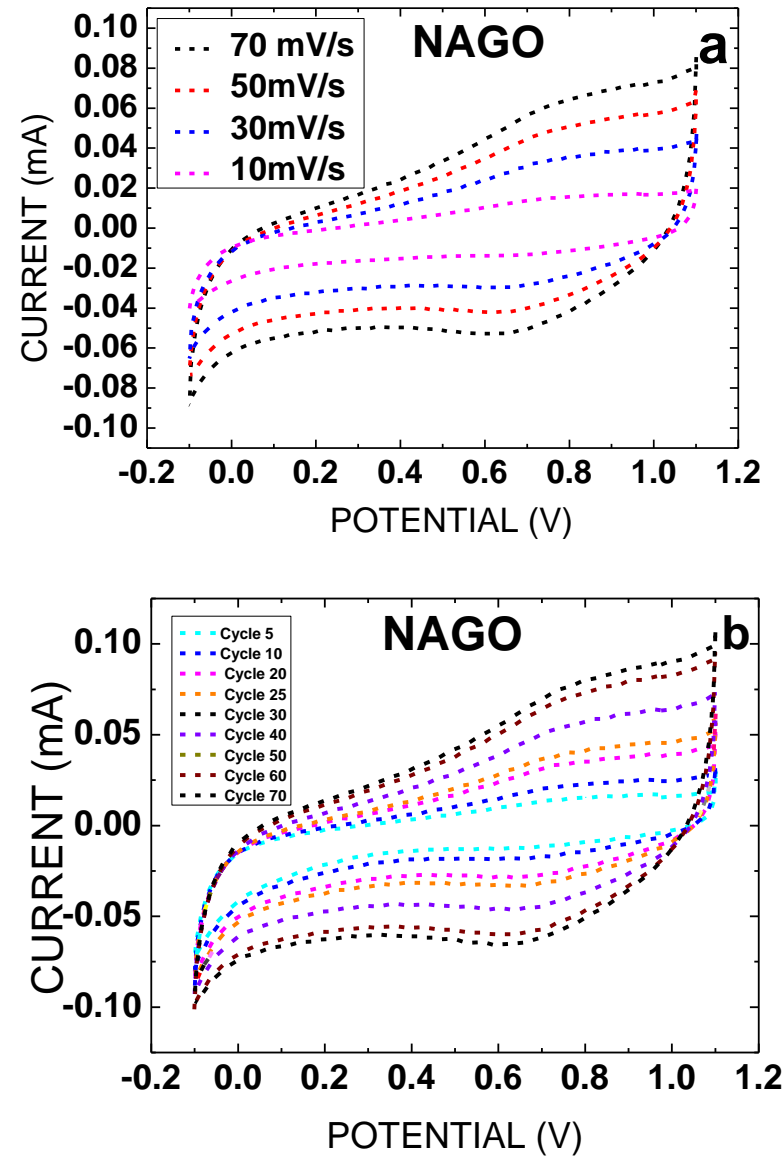

Figure 3 (a) Cyclic voltammograms (CVs) of NAGO at different scan rates. (b) Cyclic voltammograms of NAGO for different cycle number at scan rate. 


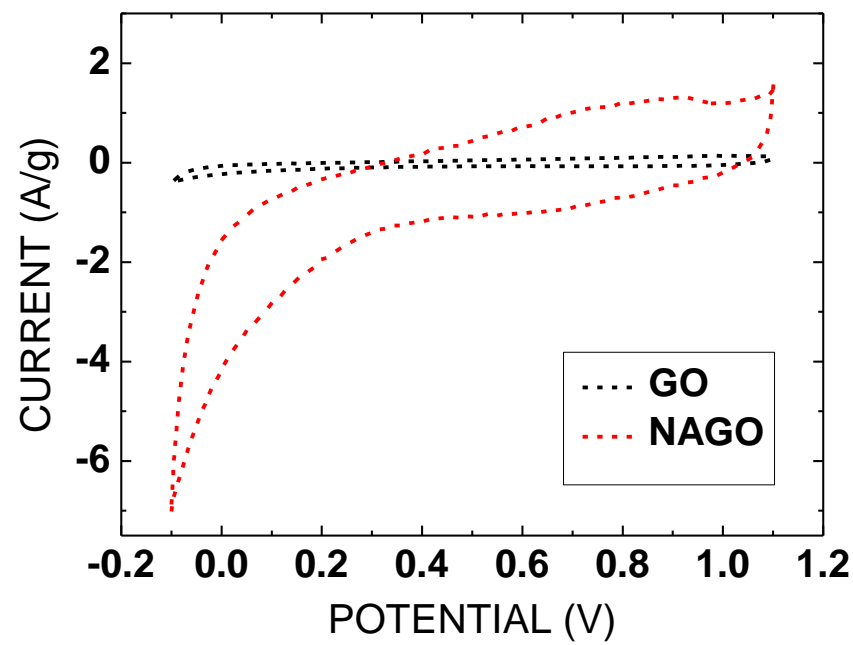

Figure 4 Cyclic voltammogram for NAGO and GO at scan rate of $100 \mathrm{mV} / \mathrm{s}$

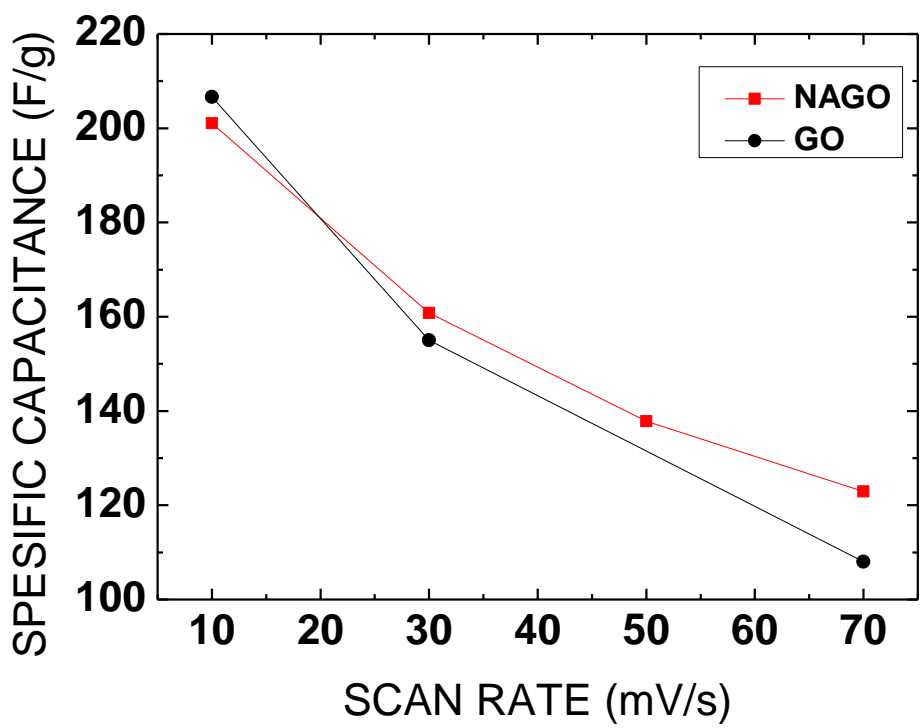

Figure 5 The maximum specific capacitance of GO and NAGO.

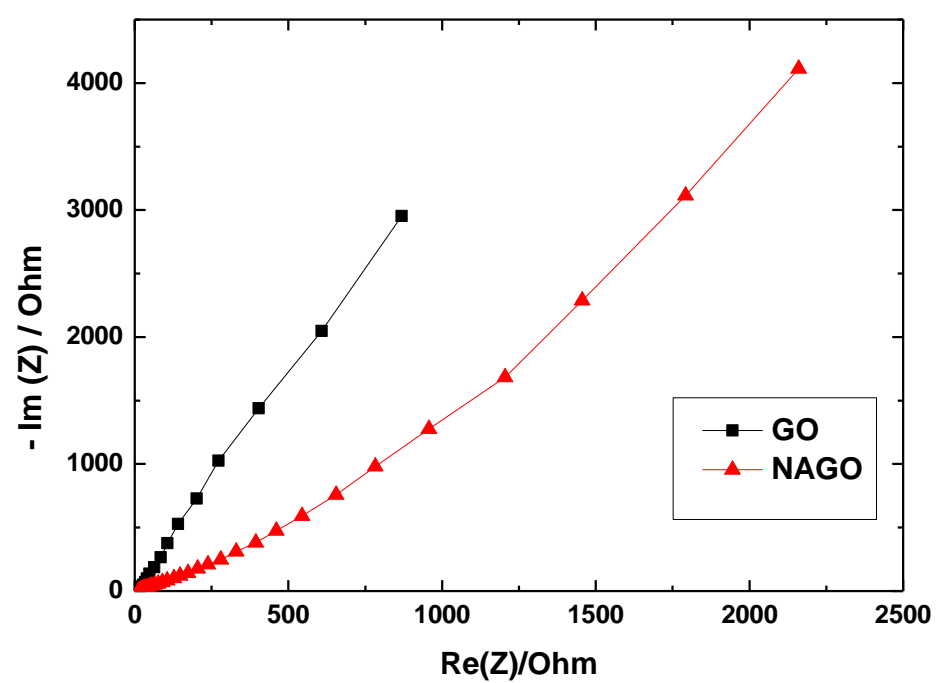

Figure 6 Nyquist plot for $\mathrm{GO}$ and TRGO in $1 \mathrm{M} \mathrm{H}_{2} \mathrm{SO}_{4}$ and $0.5 \mathrm{~V}$ at room temperature in frequency range $200 \mathrm{kHz}-0.1 \mathrm{~Hz}$ 


\section{Conclusion}

High temperature annealing of GO nanosheets were performed at $950^{\circ} \mathrm{C}$ in nitrogen ambient. Predominate exfoliated typical hexagonal layers of GO were observed by HRTEM which reflects the ideal structure of GO and hundred nanometers few layers of NAGO were obtained. According to the results in this paper, an obviousincrease in the current after reduction is attributed to the electro-catalytic activity of NAGO.

The enhanced capacitance and low impedance of NAGO is attributed to the high effective surface area and good electronic conductivity. It was demonstrated that NAGO exhibited distinguishable electrochemical performance compared to GO nanosheets.

\section{Acknowledgements}

The authors gratefully acknowledge El-Shazly M. Duraia for fruitful discussion.

\section{REFERENCES}

[1] Xiao Chen Dong, Peng Wang, Peng Chen, Carbon 49, 3672-3678 (2011).

[2] Hye Jin Park, Jannik Meyer, VieraSkakalova, Carbon48, 1088-1094 (2010).

[3] J.L. Qi, W.T. Zheng, H.W. Tian, Applied Surface Science 257, 6531-6534 (2011)

[4] K.S. Novoselov, A.K. Geim, A.A. Firsov, Science 306, 666-669 (2004).

[5] H. C. Schniepp, J. L. Li, I.A. Aksay, J. Phys Chem. B 110, 8535 (2006).

[6] Siyang Liu, Ke Chen, ZhihaoBao, Applied Surface Science 258, 5299-5303 (2012).

[7] Songfeng Pei, Jinping Zhao, Hui-Ming Cheng, Carbon 48, 4466-4474 (2010).

[8] Ke-Qin Denga, Jian-hongZhoub, Xiao-Fang Li, Colloids and Surfaces B: Biointerfaces 101, 183-188 (2013).

[9] C. Botas, P.Alvarez, C. Blanco, R. Santamaria, M. Granda, P. Ares, Carbon 50, $275-82$ (2012).

[10] Dongwoo Kang and Hyeon Suk Shin, Carbon Letters 13, 39-43 (2012).

[11] O. Akhavan, M. Kalaee, A. Esfandiar, Carbon 50, 3015-3025 (2012).

[12] SangiliyandiGurunathan, Jae Woong Han, Jin-Hoi Kim, Colloids and Surfaces B: Bio-interfaces 102, 722 (2013).

[13] ParthaKhanra, Tapas Kuila, JoongHee Lee, Chemical Engineering Journal 183, 526-533(2012).

[14] Tapas Kuilaa, Saswata Bose, JoongHee Lee, Carbon 50, 914-921 (2012).

[15] Bing Zhao, Peng Liu, Weiwen Xu, Power Sources 198, 423-427 (2012).

[16] Sh. U. Yuldashev,G. N. Panin and I. V. Khvan, Jounal of ApliedPhysics 100, 13704-13708 (2006).

[17] Michael J. McAllister, JeLuen Li, Ilhan A. Aksay, Chem. Mater 19, 4396-4404 (2007).

[18] Bing Zhao, Peng Liu, Weiwen Xu, Power Sources 198, 423-427 (2012).

[19] William S. Hummers Jr, Richard E. Offeman, American Chemical Society 80, 1339-1339 (1958).

[20] Jian Ye, Haiyan Zhang, Qiyan Ran, Power Sources 212, 105-110 (2012).

[21] F.Y. Ban, S.R. Majid, H.N. Lim, Int. J. Electro. Chem. Sci. 7, 4345-4351 (2012). 